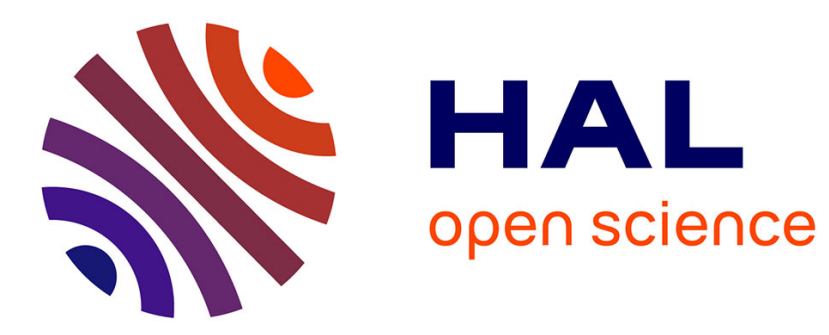

\title{
Large critical exponents for some second order uniformly elliptic operators.
}

\author{
Maria J. Esteban, Patricio Felmer, Alexander Quaas
}

\section{To cite this version:}

Maria J. Esteban, Patricio Felmer, Alexander Quaas. Large critical exponents for some second order uniformly elliptic operators. Communications in Partial Differential Equations, 2007, 32 (4), pp.543556. hal-00012841

\section{HAL Id: hal-00012841 \\ https://hal.science/hal-00012841}

Submitted on 28 Oct 2005

HAL is a multi-disciplinary open access archive for the deposit and dissemination of scientific research documents, whether they are published or not. The documents may come from teaching and research institutions in France or abroad, or from public or private research centers.
L'archive ouverte pluridisciplinaire HAL, est destinée au dépôt et à la diffusion de documents scientifiques de niveau recherche, publiés ou non, émanant des établissements d'enseignement et de recherche français ou étrangers, des laboratoires publics ou privés. 


\title{
LARGE CRITICAL EXPONENTS FOR SOME SECOND ORDER UNIFORMLY ELLIPTIC OPERATORS
}

\author{
by \\ Maria J. ESTEBAN ${ }^{a}$, Patricio L. FELMER ${ }^{b}$ \\ and \\ Alexander QUAAS ${ }^{c}$ \\ ${ }^{a}$ Ceremade UMR CNRS 7534, Université Paris-Dauphine \\ 75775 Paris Cedex 16, FRANCE. \\ ${ }^{b}$ Departamento de Ingeniería Matemática, \\ and Centro de Modelamiento Matemático, UMR2071 CNRS-UChile \\ Universidad de Chile, Casilla 170 Correo 3, \\ Santiago, CHILE. \\ ${ }^{c}$ Departamento de Matemática, Universidad Santa María, \\ Casilla: V-110, Avda. España 1680, Valparaíso, CHILE.
}

\section{Introduction}

Associated to the Laplacian we have the Sobolev critical exponent, which is the largest number $p_{N}^{*}$ having the property that the semi-linear equation

$$
\begin{aligned}
\Delta u+u^{p} & =0, \quad \text { in } \quad \Omega \\
u & =0, \quad \text { on } \partial \Omega,
\end{aligned}
$$

possesses a positive solution whenever $1<p<p_{N}^{*}$ and for any given bounded domain $\Omega$. This number, which depends on the dimension $N$, is given by $p_{N}^{*}=$ $(N+2) /(N-2)$ and its name derives from the fact that the Sobolev space $H_{0}^{1}(\Omega)$ embeds itself continuously into $L^{q}(\Omega)$, for all domain $\Omega$ of $\mathbb{R}^{N}$, if and only if $1<q \leq p_{N}^{*}+1$, and the embedding is compact if and only if $1<q<p_{N}^{*}+1$ (and if the domain is bounded or at least very small at infinity). Moreover, if $p \geq p_{N}^{*}$ and the domain is star-shaped, Pohozaev's identity implies that the above equation does not have a positive solution (see [21]). The Sobolev exponent has a dual property if the domain is $\mathbb{R}^{N}$, actually the equation has a positive solution whenever $p \geq p_{N}^{*}$ and it does not have a solution if $1<p<p_{N}^{*}$. 
If we replace the Laplacian by any linear second order uniformly elliptic operator with $C^{1}$ coefficients, say $L u=\sum_{i} \sum_{j} a_{i j} \frac{\partial u^{2}}{\partial x_{i} \partial x_{j}}$ with $a_{i j} \in C^{1}$, then the semi-linear problem

$$
\begin{aligned}
L u+u^{p} & =0, \quad \text { in } \quad \Omega \\
u & =0, \quad \text { on } \partial \Omega,
\end{aligned}
$$

has a positive solution for the same range of values of $p$, namely $1<p<p_{N}^{*}$. That is, the existence property of the Sobolev exponent remains valid for all operators in this class.

In this note we consider two classes of uniformly second order elliptic operators for which the critical exponents are drastically changed with respect to $p_{N}^{*}$, in the case of radially symmetric solutions. Our aim is to prove that the corresponding existence property for these critical exponents persists when the domain is perturbed.

Our first class corresponds to the so-called Pucci's extremal operators [19], 20] and [5]. Given positive numbers $0<\lambda \leq \Lambda$ we consider the operator $\mathcal{M}_{\lambda, \Lambda}^{+}\left(D^{2} u\right)$ where for any $N \times N$ symmetric matrix $M$,

$$
\mathcal{M}_{\lambda, \Lambda}^{+}(M)=\Lambda \sum_{e_{i}>0} e_{i}+\lambda \sum_{e_{i}<0} e_{i}
$$

$e_{i}=e_{i}(M)$ being $M$ 's eigenvalues. The case of the operator $\mathcal{M}_{\lambda, \Lambda}^{-}\left(D^{2} u\right)$ is also considered and it is defined by exchanging the roles between $\lambda$ and $\Lambda$ above.

Pucci's extremal operators appear in the context of stochastic control when the diffusion coefficient is a control variable, see the book of Bensoussan and J.L. Lions [1] or the papers of P.L. Lions [16], [17], [18] for the relation between a general Hamilton-Jacobi-Bellman and stochastic control. They also provide natural extremal equations in the sense that if $F$ is any (from linear to fully nonlinear) uniformly elliptic operator, with ellipticity constants $\lambda, \Lambda$, and depends only on the Hessian $D^{2} u$, then

$$
\mathcal{M}_{\lambda, \Lambda}^{-}(M) \leq F(M) \leq \mathcal{M}_{\lambda, \Lambda}^{+}(M)
$$

for any symmetric matrix $M$. Moreover, these operators are also extremal with respect to the first half eigenvalue of all second order elliptic operators with constant coefficients and ellipticity constants between $\lambda$ and $\Lambda$ (see for instance 任).

It is obvious that when $\lambda=\Lambda$, then $\mathcal{M}_{\lambda, \Lambda}^{ \pm}$coincides with a multiple of the Laplace operator. We also notice that given any number $s \in[\lambda, \Lambda]$ the operator $s \Delta$ belongs to the class defined by (1.5).

The second family of operators that we consider are defined as

$$
Q_{\lambda, \Lambda}^{+} u=\lambda \Delta u+(\Lambda-\lambda) Q^{0} u
$$


where $Q^{0}$ is the second order operator

$$
Q^{0} u=\sum_{i=1}^{N} \sum_{j=1}^{N} \frac{x_{i} x_{j}}{|x|^{2}} \frac{\partial^{2} u}{\partial x_{i} \partial x_{j}} .
$$

These operators are also considered by Pucci [19], being extremal with respect to some spectral properties. We notice that these operators belong to the class defined by (1.5) and when $\lambda=\Lambda$ they also become a multiple of the Laplacian. If we interchange the role of $\lambda$ by $\Lambda$ in definition (1.6), then we obtain the operator $Q_{\lambda, \Lambda}^{-}$, which is also considered later.

The operators $\mathcal{M}_{\lambda, \Lambda}^{ \pm}$are autonomous, but not linear, even if they enjoy some properties of the Laplacian. The operators $Q_{\lambda, \Lambda}^{ \pm}$are still linear, but their coefficients are not continuous at the origin. In both cases, when one considers a ball and the set of radially symmetric functions in it, there are critical exponents for the operators $\mathcal{M}^{+}$and $Q^{+}$which are greater than the Sobolev exponent $p_{N}^{*}$. On the contrary, for the operators $Q_{\lambda, \Lambda}^{-}$and $\mathcal{M}_{\lambda, \Lambda}^{-}$, the critical exponents for radially symmetric solutions in a ball are smaller than the Sobolev exponent $p_{N}^{*}$. These facts where proved in 11, 10] for $\mathcal{M}_{\lambda, \Lambda}^{ \pm}$and for $Q_{\lambda, \Lambda}^{+}$and $Q_{\lambda, \Lambda}^{-}$the proof is given here, in Section 2.

More precisely, in the case of operators $Q_{\lambda, \Lambda}^{+}$, there exists a number $\widetilde{N}_{+}=$ $\frac{\lambda}{\Lambda}(N-1)+1$, such that if $\Omega$ is a ball of $\mathbb{R}^{N}$ and if $\widetilde{N}_{+}>2$, (1.3)-(1.4) has a unique positive radially symmetric solution for any $1<p<\left(\widetilde{N}_{+}+2\right) /\left(\widetilde{N}_{+}-2\right)$ and no positive radially symmetric solution for $p \geq\left(\widetilde{N}_{+}+2\right) /\left(\widetilde{N}_{+}-2\right)$. Notice that for any $\lambda<\Lambda, \widetilde{N}_{+}<N$ and so, the critical exponent here is strictly larger than the Sobolev critical exponent $p_{N}^{*}$.

In the case of the Pucci's extremal operators $\mathcal{M}_{\lambda, \Lambda}^{+}$, the critical exponent is a number $p_{+}^{*}$ such that

$$
p_{N}^{*}<p_{+}^{*}<\frac{\widetilde{N}_{+}+2}{\widetilde{N}_{+}-2} .
$$

The number $p_{+}^{*}$ depends on $\lambda, \Lambda$ and the dimension $N$, however an explicit formula for it is not known.

Similarly, for the operator $Q_{\lambda, \Lambda}^{-}$we may also define a dimension like number $\widetilde{N}_{-}=\frac{\Lambda}{\lambda}(N-1)+1$, so that its critical exponent in the radially symmetric case is precisely $\left(\widetilde{N}_{-}+2\right) /\left(\widetilde{N}_{-}-2\right)$. In the case of the operator $\mathcal{M}_{\lambda, \Lambda}^{-}$we recall that the critical exponent of the operator $\mathcal{M}_{\lambda, \Lambda}^{-}$is a number $p_{-}^{*}$ satisfying

$$
\frac{\tilde{N}_{-}+2}{\widetilde{N}_{-}-2}<p_{-}^{*}<p_{N}^{*},
$$

as it was shown in [11].

It is the purpose of this note to prove that this phenomenom of critical exponent increase (or decrease) does not appear only in the radially symmetric case. By a 
perturbation argument, based on a work by Dancer [9], we show that these critical exponents, with respect to existence properties in bounded domains, persist when the ball is perturbed not necessarily in a radial manner. This result provides us with evidence that the critical exponents for these operators, obtained in radial versions, are also the critical exponents in the general case.

At this point we would like to stress some surprising properties of the critical exponents of operators in the class given by (1.5). For the first property we consider all linear elliptic operators with bounded coefficients and belonging to the class defined by (1.5). If we take the $L^{\infty}$ topology for the coefficients of these operators, we see that the critical exponent is not a continuous function of the operator. In particular, as shown in Section 2, the operators $Q_{\lambda, \Lambda}^{ \pm}$can be "approximated" in $L^{\infty}$ (the coefficients) by a sequence of operators with $C^{\infty}$ coefficients, for which the critical exponent in the radially symmetric case is $p_{N}^{*}$.

The second property is related to the non-monotonicity of the critical exponents. Notice the following operator's inequalities,

$$
\lambda \Delta \leq \mathcal{M}_{\lambda, \Lambda}^{+} \quad \text { and } \quad Q_{\lambda, \Lambda}^{+} \leq \mathcal{M}_{\lambda, \Lambda}^{+}
$$

while for the corresponding critical exponents we have

$$
p_{*}^{N}<p_{+}^{*} \quad \text { and } \quad \frac{\widetilde{N}_{+}+2}{\widetilde{N}_{+}-2}>p_{+}^{*} .
$$

We finally observe that all operators of the form $\mathcal{M}_{s, S}^{ \pm}$and $Q_{s, S}^{ \pm}$, with $s, S \in$ $[\lambda, \Lambda]$, have critical exponents in the interval

$$
\left[\begin{array}{cc}
\frac{\tilde{N}_{-}+2}{\widetilde{N}_{-}-2}, & \frac{\widetilde{N}_{+}+2}{\widetilde{N}_{+}-2}
\end{array}\right] .
$$

We conjecture that in the class of operators defined by (1.5), the critical exponents are all in the same interval, that is, the operators $Q_{\lambda, \Lambda}^{ \pm}$are extremal for critical exponents.

This is article is organized in two sections. In section 2 we discuss the case of the operator $Q_{\lambda, \Lambda}^{+}$. We first consider the radial case analyzing, in analogy with the case of the Laplacian, the critical exponent and proving that in the subcritical case the positive solution is non-degenerate. Then, by linearization, we show that this solution is non-degenerate in the space of functions not necessarily symmetric and we apply a perturbation argument via degree theory. In Section 3 we consider the case of the operator $\mathcal{M}_{\lambda, \Lambda}^{+}$. The situation here is somehow simpler since we know that in the ball all positive solutions are radially symmetric by using a moving plane argument, and then, in the subcritical case, the radial solution is isolated. We conclude by a homotopy invariance with respect to the ellipticity constant, as in 洒 and then by using a perturbation argument. 


\section{The extremal operator $Q_{\lambda, \Lambda}^{+}$.}

In this section we analyze the equation (1.3)-(1.4) in the case of the extremal operator $Q_{\lambda, \Lambda}^{+}$, with $0<\lambda<\Lambda$. This is a uniformly elliptic operator, whose coefficients have a discontinuity at the origin. This feature is what makes this operator interesting. We need to make precise the very notion of a solution for equation (1.3)-(1.4).

We observe that the operator $Q^{0}$ corresponds to the second derivative with respect to the radial coordinate $r=|x|$, that is

$$
Q^{0} u=\frac{\partial^{2} u}{\partial r^{2}}
$$

Because of this observation, we see that the analysis of the radial case is very simple, it amounts to change the notion of the dimension taking into account $\lambda$ and $\Lambda$, and to perform a phase plane analysis. In fact, we easily see that if $u$ is a solution of

$$
\Lambda u^{\prime \prime}+\lambda \frac{N-1}{r} u^{\prime}+u^{p}=0, \quad u^{\prime}(0)=u(1)=0 .
$$

then $u(x)=u(|x|)$ is a solution of (1.3)-(1.4). Defining

$$
\widetilde{N}_{+}=\frac{\lambda}{\Lambda}(N-1)+1 \quad \text { and } \quad v(r)=(\Lambda)^{1 /(1-p)} u(r)
$$

we see that $v$ satisfies

$$
v^{\prime \prime}+\frac{\tilde{N}_{+}-1}{r} v^{\prime}+v^{p}=0, \quad v^{\prime}(0)=v(1)=0 .
$$

The following theorem gives the critical exponent for equation (2.2) and the nondegeneracy property of its solutions in the subcritical case.

Theorem 2.1 Equation (2.8) does not have a positive solution if

$$
p \geq\left(\tilde{N}_{+}+2\right) /\left(\tilde{N}_{+}-2\right),
$$

and it possesses exactly one positive solution if

$$
1<p<\left(\tilde{N}_{+}+2\right) /\left(\tilde{N}_{+}-2\right) \quad \text { and } \quad \tilde{N}_{+}>2
$$

or

$$
1<p \quad \text { and } \quad 1<\tilde{N}_{+} \leq 2 .
$$

Moreover, if $v$ is a solution of (2.8), then the linearized equation

$$
h^{\prime \prime}+\frac{\widetilde{N}_{+}-1}{r} h^{\prime}+p v^{p-1} h=0, \quad h^{\prime}(0)=h(1)=0,
$$

has no non-trivial solution, that is, 0 is not in the spectrum of the linearized operator. 
Proof. The criticality of the number $\left(\widetilde{N}_{+}+2\right) /\left(\widetilde{N}_{+}-2\right)$ can be proved in a way similar to the case of the Laplacian, by using the Emden-Fowler transformation. Let $v$ be a positive solution of equation (2.2), then we have that

$$
v_{\gamma}(r)=\gamma v\left(\gamma^{(p-1) / 2} r\right)
$$

also satisfies the equation in (2.2) together with boundary conditions $v_{\gamma}^{\prime}(0)=0$ and $v_{\gamma}\left(\gamma^{(1-p) / 2}\right)=0$, for all positive $\gamma$. From here we see that the function

$$
h_{1}(r)=\left.\frac{\partial v_{\gamma}}{\partial \gamma}(r)\right|_{\gamma=1},
$$

satisfies $(2.3)$ and $h_{1}^{\prime}(0)=0, h_{1}(0)>0$.

Assume that $h_{2}$ is a second solution, linearly independent of $h_{1}$. Then necessarily we have that $h_{2}^{\prime}(r)$ stays away from zero, for $r$ near 0 , since the contrary implies that $h_{1}$ and $h_{2}$ are linearly dependent. Now, given any solution $h$ of (2.3), we have $h=c_{1} h_{1}+c_{2} h_{2}$. But then $c_{2}=0$ since $h^{\prime}(0)=0$ and $c_{1}=0$ since $h_{1}(1)<0$, proving that $h \equiv 0$.

Continuing with our analysis, we observe that since the operator $Q^{0}$, and then also $Q_{\lambda, \Lambda}^{+}$, has discontinuous coefficients, we should start making precise the notion of solution for the equation

$$
\begin{aligned}
& Q_{\lambda, \Lambda}^{+} u=f \text { in } \Omega, \\
& u=0 \quad \text { on } \partial \Omega \text {. }
\end{aligned}
$$

For notational simplicity, in the rest of this section we simply write $Q$ for $Q_{\lambda, \Lambda}^{+}$, since no confusion will arise.

Given $i, j$ we consider a sequence of $C^{\infty}$ functions $a_{i, j}^{n}$ so that $a_{i, j}^{n}(x)=$ $x_{i} x_{j} /|x|^{2}$ for all $|x| \geq 1 / n$ and $\left|a_{i, j}^{n}(x)\right| \leq 1$ for $|x| \leq 1 / n$. For example, we may consider a cut-off function $\eta$ so that $\eta(r)=0$ if $r<1 / 2$ and $\eta(r)=1$ if $r \geq 1$, and then define

$$
a_{i, j}^{n}(x)=\eta(n r) \frac{x_{i} x_{j}}{|x|^{2}} .
$$

Then we define the operators

$$
Q^{n} u=\lambda \Delta u+(\Lambda-\lambda) \sum_{i=1}^{n} \sum_{j=1}^{n} a_{i, j}^{n} \frac{\partial^{2} u}{\partial x_{i} \partial x_{j}}
$$

We assume that the function $f$ is continuous in $\bar{\Omega}$, then the problem

$$
\begin{aligned}
Q^{n} u=f & \text { in } \quad \Omega, \\
u=0 & \text { on } \partial \Omega,
\end{aligned}
$$


possesses a unique smooth solution $u_{n}$. Moreover, since the coefficients are $C^{\infty}$ functions it is well known the existence of a Green function $G_{n}: \Omega \times \Omega \rightarrow \mathbb{R}$ allowing to represent this solution as

$$
u_{n}(x)=\int_{\Omega} G_{n}(x, y) f(y) d y .
$$

It follows from Alexandrof-Bakelman-Pucci's estimate that the sequence $\left\{G_{n}(x, \cdot)\right\}$ is bounded in $L^{N /(N-1)}(\Omega)$ and hence, up to subsequence, it has a weak limit in this space. Moreover, since our operator $Q$ is discontinuous just at one point, the origin, the weak limit is unique as shown by the arguments in Cerutti, Escauriaza and Fabes [7]. Thus, our problem has a unique Green function $G: \Omega \times \Omega \rightarrow \mathbb{R}$, such that $G(x, \cdot)$ is in $L^{N /(N-1)}(\Omega)$, and we define

$$
u(x)=\int_{\Omega} G(x, y) f(y) d y,
$$

as the solution to equation (2.4) and (2.5).

On the other hand, the sequence of solutions $\left\{u_{n}\right\}$ is bounded in $C_{0}^{\alpha}(\bar{\Omega})$, for $\alpha>0$ as follows from basic estimates, see Gilbarg and Trudinger 13] or Cabré Caffarelli [5]. Then, the solution $u$ is actually of class $C^{\alpha}(\bar{\Omega})$ and we have

$$
\|u\|_{C^{\alpha}(\bar{\Omega})} \leq C\|u\|_{L^{\infty}(\partial \Omega)}+C\|f\|_{L^{N}(\Omega)} .
$$

Here the constant $C$ depends only on the ellipticity constants, the $L^{\infty}$ bounds on the coefficients and on the domain $\Omega$, which we assume to have a regular boundary.

Let $u_{0}$ be the unique solution of (2.1). In what follows we show that, when $u_{0}$ is considered as a function of the $N$ variables, it satisfies the equation in the sense given above.

Lemma 2.1 The function $u_{0}(x)=u_{0}(|x|)$ satisfies the equation

$$
\begin{aligned}
Q u & =-u^{p} \text { in } B, \\
u & =0 \text { on } \partial B,
\end{aligned}
$$

in the sense just defined above.

Proof. By direct computation we see that, pointwise, we have

$$
Q^{n} u=-u^{p}-c_{n}(x) \text { in } B,
$$

where $c_{n}$ is a function with support in the ball $B(0,1 / n)$ and which is bounded, with a bound independent of $n$. Then we certainly have

$$
u(x)=\int_{B} G_{n}(x, y)\left(-u^{p}(y)+c_{n}(y)\right) d y .
$$

Taking limits here we conclude. 
Remark 2.1 The notion of solution defined above is known as good solution and it was introduced by Cerutti, Escauriaza and Fabes [7]. In a recent paper by Jensen, Kocan and Swiech [15], this notion of solutions is shown to be equivalent to $L^{p}$ viscosity solution.

Remark 2.2 We do not know whether equation (2.10)-(2.11) possesses a nonradial solution or not.

Our existence result is for domains which are close to the unit ball. More precisely we assume that we have a sequence of domains $\left\{\Omega_{n}\right\}$ such that for all $0<r<1<R$ there exists $n_{0} \in \mathbb{N}$ such that

$$
B(0, r) \subset \Omega_{n} \subset B(0, R), \quad \text { for all } n \geq n_{0} .
$$

We consider a reference bounded domain $D$ such that $\Omega_{n} \subset D$, for all $n \in \mathbb{N}$. We may take, for example, $D$ as the ball of radius 2 .

Next we prove a continuity property for the Green functions associated to the domains $\Omega_{n}$

Lemma 2.2 Under the conditions given above we have, for every $f \in C(D)$ and for every $x \in B$,

$$
\lim _{n \rightarrow \infty} \int_{\Omega_{n}} G_{\Omega_{n}}(x, y) f(y) d y=\int_{B} G_{B}(x, y) f(y) d y,
$$

where $G_{B}$ and $G_{\Omega_{n}}$ are the Green functions of the unit ball $B$ and of $\Omega_{n}$, respectively.

Proof. Let $f \in C(D)$ and let $u_{n}$ be the solution of the equation

$$
Q u=f, \quad \text { in } \quad \Omega_{n}, \quad u=0 \quad \text { on } \quad \partial \Omega_{n} .
$$

Consider also the function $u$, the solution of the equation

$$
Q u=f, \quad \text { in } \quad B_{R}, \quad u=0 \quad \text { on } \partial B_{R} .
$$

Here we assume $r<1<R$ are close to 1 and $n$ is large enough so that $B_{r} \subset \Omega_{n} \subset$ $B_{R}$. Using (2.9) we find

$$
\|u\|_{C^{\alpha}\left(\bar{B}_{R}\right)} \leq C\|f\|_{L^{N}\left(B_{R}\right)}
$$

which implies

$$
|u(x)| \leq C(R-r)^{\alpha}\|f\|_{L^{N}\left(B_{R}\right)}, \quad x \in B_{R} \backslash B_{r} .
$$


Since $u_{n}-u$ satisfies $Q\left(u_{n}-u\right)=0$ in $\Omega_{n}$, by standard estimates, see [5], we obtain

$$
\left\|u_{n}-u\right\|_{L^{\infty}\left(\Omega_{n}\right)} \leq C\left\|u_{n}-u\right\|_{L^{\infty}\left(\partial \Omega_{n}\right)} \leq C(R-r)^{\alpha}\|f\|_{L^{N}\left(B_{R}\right)},
$$

where the constant $C$ here is uniform in $n$. Similarly if $v$ is the solution of

$$
Q u=f, \quad \text { in } B, \quad u=0 \quad \text { on } \quad \partial B
$$

then we have

$$
\|v-u\|_{L^{\infty}(B)} \leq C(R-r)^{\alpha}\|f\|_{L^{N}(B)} .
$$

Since for all $x \in B_{r}$ we have

$$
u_{n}(x)-v(x)=\int_{\Omega_{n}} G_{\Omega_{n}}(x, y) f(y)-\int_{B} G_{B}(x, y) f(y),
$$

the result follows.

Now we state and prove our main theorem of this section.

Theorem 2.2 Assume $\tilde{N}_{+}>2$ and that $1<p<\left(\tilde{N}_{+}+2\right) /\left(\tilde{N}_{+}-2\right)$. Then there is $n_{0} \in \mathbb{N}$ so that for all $n \geq n_{0}$, the equation

$$
\begin{aligned}
Q u+u^{p} & =0 \text { in } \Omega_{n}, \\
u & =0 \text { on } \partial \Omega_{n},
\end{aligned}
$$

possesses at least one nontrivial solution.

In order to prove our theorem we will follow some ideas from [9]. We start setting up the functional analytic framework. We consider the inclusions $i: C_{0}(\bar{D}) \rightarrow$ $C(\bar{B})$ and $j: C_{0}^{\alpha}(\bar{D}) \rightarrow C_{0}(\bar{D})$. Here $C$ stands for continuous functions, $C_{0}$ for continuous functions vanishing on the boundary and $C_{0}^{\alpha}$ for Hölder continuous functions vanishing on the boundary. Given $f \in C(\bar{B})$, we let $\overline{\mathcal{L}}(f)$ be the unique solution to (2.4)-(2.5) with $\Omega=B$. Then we extend this solution to define $\mathcal{L}(f)$ as

$$
\mathcal{L}(f)(x)=\left\{\begin{array}{lll}
\overline{\mathcal{L}}(f)(x) & \text { if } \quad x \in \bar{B} \\
0 & \text { if } \quad x \in \bar{D} \backslash B
\end{array}\right.
$$

Thus, the operator $\mathcal{L}: C(\bar{B}) \rightarrow C_{0}^{\alpha}(\bar{D})$ is well defined as a linear bounded operator. Next we define the nonlinear operator $\mathcal{F}: C_{0}(\bar{D}) \rightarrow C_{0}(\bar{D})$ as

$$
\mathcal{F}(u)=-j \circ \mathcal{L}\left(i\left(u^{p}\right)\right) .
$$

If we consider $N: C_{0}(\bar{D}) \rightarrow C_{0}(\bar{D})$, the Nemitsky operator defined as $N(u)=u^{p}$, we easily see that $N$ is of class $C^{1}$ and $N^{\prime}(u)(h)=p u^{p-1} h$ for $h \in C_{0}(\bar{D})$. Thus, the operator $\mathcal{F}$ is compact and of class $C^{1}$.

In the definition of the operators $i$ and $\mathcal{L}$ we can replace $B$ by $\Omega_{n}$ and we obtain $i_{n}$ and $\mathcal{L}_{n}$. The operator $\mathcal{F}_{n}$ is then defined as $\mathcal{F}_{n}(u)=-j \circ \mathcal{L}_{n}\left(i_{n}\left(u^{p}\right)\right)$, and naturally it is compact and of class $C^{1}$ as an operator in $C_{0}(\bar{D})$. 
Before giving the proof of Theorem 2.2 we need still another preliminary result. It guarantees that $u_{0}$ is isolated not only in the space of radial functions, as shown in Theorem 2.1, but also in the space of all functions of class $C^{\alpha}$ in the ball $B=B(0,1)$.

Proposition 2.1 Under the hypotheses of Theorem 2.9, the linear equation

$$
\begin{aligned}
Q h+p u_{0}^{p-1} h & =0 \quad \text { in } \quad B, \\
h & =0 \quad \text { on } \partial B,
\end{aligned}
$$

has only the trivial solution $h \equiv 0$ in $C_{0}^{\alpha}(B)$.

Proof. Our proof uses a standard argument by Smoller and Wasserman 23]. We let $\left\{\phi_{k}(\theta)\right\}_{k=0}^{\infty}, \theta \in S^{N-1}$, be the eigenfunctions of the Laplacian in $S^{N-1}$, whose eigenvalues are

$$
\lambda_{k}=-k(k+N-2) .
$$

Assume $h$ is a solution of (2.16)-(2.17). Then we consider the approximation operator $Q^{n}$ as given by (2.6) and we solve the equation

$$
Q^{n} h_{n}=-p u_{0}^{p-1} h \quad \text { in } B, \quad h_{n}=0, \quad \text { on } \partial B .
$$

We observe that if $u \in C^{2}$ and $u=u(r, \theta)$ with $\theta \in S^{N-1}$ then for the operator $Q^{n}$ we can write

$$
Q^{n} u=\{\lambda+(\Lambda-\lambda) \eta(n r)\} u^{\prime \prime}+\lambda \frac{N-1}{r} u^{\prime}+\frac{\lambda}{r^{2}} \Delta_{\theta} u,
$$

where' denotes derivative with respect to $r$ and $\Delta_{\theta}$ is the Laplacian on the sphere. Let us define

$$
a_{k}^{n}(r)=\int_{S^{N-1}} h_{n} \phi_{k} d \theta \quad \text { and } a_{k}(r)=\int_{S^{N-1}} h \phi_{k} d \theta .
$$

By the $C^{\alpha}$ convergence of $h_{n}$ to $h$ we see that $a_{k}^{n}$ converges uniformly in $[0,1]$ to $a_{k}$, for all $k \geq 0$. Next we multiply equation $(2.18)$ by $\phi_{k}$ and integrate over $S^{N-1}$ to obtain

$$
\{\lambda+(\Lambda-\lambda) \eta(n r)\}\left(a_{k}^{n}\right)^{\prime \prime}+\lambda \frac{N-1}{r}\left(a_{k}^{n}\right)^{\prime}+\lambda \lambda_{k} \frac{a_{k}^{n}}{r^{2}}=-p u_{0}^{p-1} a_{k} .
$$

From here we can prove that for $r \in(0,1]$ the convergence of $a_{k}^{n}$ to $a_{k}$ is even $C^{2}$ and $a_{k}$ satisfies

$$
a_{k}^{\prime \prime}+\frac{\tilde{N}-1}{r} a_{k}^{\prime}+\frac{\lambda \lambda_{k}}{\Lambda} \frac{a_{k}}{r^{2}}+\frac{p}{\Lambda} u_{0}^{p-1} a_{k}=0 .
$$

Moreover, we can prove that $a_{k}(0)=0$ and that $r^{\tilde{N}_{+}-1} a_{k}^{\prime}(r)$ is bounded as $r \rightarrow 0$. To prove the last statement we use that $\tilde{N}_{+}>2$. On the other hand we have that $w=u_{0}^{\prime}$ satisfies the equation

$$
w^{\prime \prime}+\frac{\tilde{N}-1}{r} w^{\prime}-\frac{\tilde{N}-1}{r^{2}} w+\frac{p}{\Lambda} u_{0}^{p-1} w=0 .
$$


Multiplying equation (2.19) by $w$ and equation (2.20) by $a_{k}$, integrating between 0 and the first zero of $a_{k}$, and subtracting we can prove that $a_{k} \equiv 0$ for all $k \geq 1$, see [23]. For $k=0$ we use Theorem 2.1 to prove that also $a_{0} \equiv 0$.

We finally complete the proof of our main theorem in this section.

Proof of Theorem 2.2. Since $I-\mathcal{F}^{\prime}\left(u_{0}\right)$ has trivial kernel, as we proved in Proposition 2.1, there is $\delta>0$ such that $u-\mathcal{F}(u) \neq 0$ for all $u \in \partial \mathcal{B}$, where $\mathcal{B}=\left\{u \in C_{0}(\bar{D}) /\left\|u-u_{0}\right\|_{C_{0}(\bar{D})}<\delta\right\}$. Moreover, the Leray-Schauder degree of $I-\mathcal{F}$ is well defined in $\mathcal{B}$ and $\operatorname{deg}(I-\mathcal{F}, \mathcal{B}, 0)=1$ or -1 . To finish the proof we just need to prove that there exists $n_{0} \in \mathbb{N}$ so that

$$
u \neq t \mathcal{F}(u)+(1-t) \mathcal{F}_{n}(u), \quad \text { for all } t \in[0,1], u \in \partial \mathcal{B},
$$

since this implies that

$$
\operatorname{deg}\left(I-\mathcal{F}_{n}, \mathcal{B}, 0\right)=\operatorname{deg}(I-\mathcal{F}, \mathcal{B}, 0) \neq 0 .
$$

Let us assume that (2.21) is not true. Then there exist sequences $\left\{t_{n}\right\} \subset[0,1]$ and $\left\{u_{n}\right\} \subset \partial \mathcal{B}$ such that

$$
u_{n}=t_{n} \mathcal{F}\left(u_{n}\right)+\left(1-t_{n}\right) \mathcal{F}_{n}\left(u_{n}\right) .
$$

We may assume that $u_{n} \rightarrow \bar{u}$ uniformly in $\bar{D}$, up to a subsequence, as a consequence of the compactness of the inclusion $j$. We may also assume $t_{n} \rightarrow \bar{t}$. Then we see that we will get a contradiction if we prove that

$$
\lim _{n \rightarrow \infty} \mathcal{F}_{n}\left(u_{n}\right)=\mathcal{F}(\bar{u})
$$

From the definition of $\mathcal{F}_{n}$ we see that if $v_{n}=\mathcal{F}_{n}\left(u_{n}\right)$ then

$$
v_{n}(x)=-\int_{\Omega_{n}} G_{\Omega_{n}}(x, y) u_{n}^{p}(y) d y,
$$

where $G_{\Omega_{n}}$ is the Green function of $\Omega_{n}$. Since $u_{n}$ is uniformly convergent to $\bar{u}$ in $\bar{D}$, to complete the proof we just use Lemma 2.2 .

Remark 2.3 Our Theorem 2.2 is concerned with the operator $Q_{\lambda, \Lambda}^{+}$, for $0<$ $\lambda \leq \Lambda$. A completely analogous theorem can be proved for the operator $Q_{\lambda, \Lambda}^{-}$. Naturally, our hypotheses have to be changed to: $\tilde{N}_{-}>2$ and $1<p<\left(\tilde{N}_{-}+\right.$ 2)/( $\left.\tilde{N}_{-}-2\right)$. 


\section{The extremal operator $\mathcal{M}_{\lambda, \Lambda}^{+}$.}

In this section we analyze equation (1.3)-(1.4) in the case $L=M_{\lambda, \Lambda}^{+}$, the extremal Pucci's operator for $0<\lambda \leq \Lambda$. This is an elliptic operator which is nonlinear, but homogenous of degree 1 . In comparison with $Q_{\lambda, \Lambda}^{+}$, for the operator $\mathcal{M}_{\lambda, \Lambda}^{+}$there is a good regularity theory that guarantees that the solutions to equation (1.3)-(1.4) are classical solutions (see [5]).

We start this section recalling the existence of positive solutions for (1.3)-(1.4), when $\Omega$ is a ball as was proved by Felmer and Quaas in [10] and [11].

Theorem 3.1 Suppose $\tilde{N}_{+}>2$. Then there exist a number $p_{+}^{*}$ such that

$$
\frac{N+2}{N-2}<p_{+}^{*}<\frac{\widetilde{N}_{+}+2}{\widetilde{N}_{+}-2} .
$$

with the property that if $1<p<p_{*}^{+}$then (1.3)-(1.4) has a nontrivial radially symmetric $C^{2}$ solution. Moreover, if $p \geq p_{*}^{+}$then (1.3)-(1.4) does not have a solution.

The number $p_{*}^{+}$is called critical exponents for the operator $M_{\lambda, \Lambda}^{+}$. We notice that $\lambda=\Lambda$ implies $p_{*}^{+}=p_{*}^{N}$.

Let $\Omega_{n}$ be a sequence of domains satisfying the conditions given in the previous section. Now we present the main theorem of this section

Theorem 3.2 Assume $\tilde{N}_{+}>2$ and that $1<p<p_{+}^{*}$. Then there is $n_{0} \in \mathbb{I}$ so that for all $n \geq n_{0}$, the equation

$$
\begin{aligned}
\mathcal{M}_{\lambda, \Lambda}^{+}\left(D^{2} u\right)+u^{p} & =0 \quad \text { in } \Omega_{n}, \\
u & =0 \quad \text { on } \partial \Omega_{n},
\end{aligned}
$$

possesses at least one positive solution.

Remark 3.1 It can be seen that equation (1.3)-(1.4), when $\Omega$ is a ball, has only one positive radially symmetric solution. In fact, by using a classical moving planes technique (see [3]), all positive solutions of (1.3)-(1.4) are radially symmetric (for detail see [8]). This uniqueness property is crucial in our analysis, since it allows us to use a degree theory approach as in the previous section, avoiding the study of the linearized equation in order to obtain non-degeneracy of the radially symmetric solution.

Remark 3.2 a) Our Theorem 3.2 is concerned with the operator $M_{\lambda, \Lambda}^{+}$, for $0<$ $\lambda \leq \Lambda$. A completely analogous theorem can be proved for the operator $M_{\lambda, \Lambda}^{-}$. With the natural change in the hypothesis to $1<p<p_{-}^{*}$.

b) For other existence result concerning Pucci operator we refer the reader to [12] and [22]. 
Proof. Let $q:[\lambda, \Lambda] \rightarrow \mathbf{R}$ be a continuous function such that $q(s)<p_{*}^{+}(s)$ for all $s \in[\lambda, \Lambda]$ and $q(\lambda)=p$. Here $p_{*}^{+}(s)$ is the critical exponent for the operator $\mathcal{M}_{s, \Lambda}^{+}$, which is a continuous function of $s$. Next we consider $s \in[\lambda, \Lambda]$ and we define $\overline{\mathcal{L}}^{s}(f)$ as the unique solution to

$$
\begin{aligned}
\mathcal{M}_{s, \Lambda}^{+}\left(D^{2} u\right) & =f \quad \text { in } B, \\
u & =0 \quad \text { on } \partial B,
\end{aligned}
$$

for $f \in C(\bar{B})$. This operator is well defined in $C(\bar{B})$, with values in $C_{0}^{\alpha}(\bar{B})$, and it is positive, that is if $f(x) \leq 0$ in $B$ then $u \geq 0$ in $B$. These follow from existence and regularity theory for fully nonlinear operators and the maximum principle for $\mathcal{M}_{s, \Lambda}^{+}$. See the monography by Caffarelli and Cabré [5] and the work by Bardi and Da Lio [2]. Now we extend $\overline{\mathcal{L}}^{s}$ as in (2.14) and define the operator $\mathcal{F}(s, \cdot)$ using (2.15), with $p=q(s)$.

Let $u_{0}^{s}$ be the unique solution of (1.3)-(1.4) in $B$ for $p=q(s)$ given by Theorem 3.1. We consider

$$
M=\sup _{s \in[\lambda, \Lambda]}\left\|u_{0}^{s}\right\|_{L^{\infty}(B)} \quad \text { and } \quad m=\inf _{s \in[\lambda, \Lambda]}\left\|u_{0}^{s}\right\|_{L^{\infty}(B)}>0
$$

and let $\delta>0$ be such that $m-\delta>0$. We define the set

$$
\mathcal{B}=\left\{u \in C_{0}(\bar{D}) / u(x) \geq 0 \text { in } D, m-\delta<\|u\|_{C_{0}(\bar{D})}<M+1\right\},
$$

and we observe, as follows by the uniqueness of solutions in the ball and the strong maximum principle for $\mathcal{M}_{s, \Lambda}^{+}$, that $u-F(s, u) \neq 0$ for all $u \in \partial \mathcal{B}$ and $s \in[\lambda, \Lambda]$.

We notice that $\operatorname{deg}(I-\mathcal{F}(\Lambda, \cdot), \mathcal{B}, 0) \neq 0$, since in this case we are dealing with the Laplacian. Then by invariance under homotopy of the degree we obtain

$$
\operatorname{deg}(I-\mathcal{F}(\lambda, \cdot), \mathcal{B}, 0)=\operatorname{deg}(I-\mathcal{F}(\Lambda, \cdot), \mathcal{B}, 0) \neq 0
$$

Next we define $\mathcal{F}_{n}$ perturbing the domain as in the previous section, keeping $s=\lambda$ and $p=q(\lambda)$. To finish the proof we just need to prove that there exists $n_{0} \in \mathbb{N}$ so that for all $n \geq n_{0}$

$$
u \neq t \mathcal{F}(\lambda, u)+(1-t) \mathcal{F}_{n}(\lambda, u), \quad \text { for all } \quad t \in[0,1], u \in \partial \mathcal{B},
$$

since this implies that

$$
\operatorname{deg}\left(I-\mathcal{F}_{n}(\lambda, \cdot), \mathcal{B}, 0\right)=\operatorname{deg}(I-\mathcal{F}(\lambda, \cdot), \mathcal{B}, 0) \neq 0
$$

Let us assume that (3.5) is not true. Then there exist sequences $\left\{t_{n}\right\} \subset[0,1]$ and $\left\{u_{n}\right\} \subset \partial \mathcal{B}$ such that

$$
u_{n}=t_{n} \mathcal{F}\left(\lambda, u_{n}\right)+\left(1-t_{n}\right) \mathcal{F}_{n}\left(\lambda, u_{n}\right)
$$


We may assume that $t_{n} \rightarrow \bar{t}$ and that $u_{n} \rightarrow \bar{u}$ uniformly in $\bar{D}$, up to a subsequence, as a consequence of the compactness of the inclusion $j$. We will get a contradiction if we prove that

$$
\lim _{n \rightarrow \infty} \mathcal{F}_{n}\left(\lambda, u_{n}\right)(x)=\mathcal{F}(\lambda, \bar{u})(x) \quad \text { for all } x \in D
$$

We first notice that $\mathcal{F}_{n}\left(\lambda, u_{n}\right):=v_{n} \rightarrow v$ in $C_{0}(D)$. If $x \in D \backslash \bar{B}$, then for $n$ large $\mathcal{F}_{n}\left(\lambda, u_{n}\right)(x)=0$, and so $v(x)=0$, therefore by continuity of $v$ we also have $v=0$ in $D \backslash B$. On the other hand

$$
\mathcal{M}_{\lambda, \Lambda}^{+}\left(D^{2} v_{n}\right)=-u_{n}^{p} \quad \text { in } \quad B(0, r)
$$

for $n$ large. Passing to the limit in the viscosity sense we get $\mathcal{M}_{\lambda, \Lambda}^{+}\left(D^{2} v\right)=-\bar{u}^{p}$ in $B$. Since $v=0$ on $\partial B$ then, by the definition of $\mathcal{F}$, we obtain $\mathcal{F}(\lambda, \bar{u})=v$, concluding the proof.

Acknowledgements The second author was partially supported by Fondecyt Grant \# 1030929 and FONDAP de Matemáticas Aplicadas. The third author was partially supported by Fondecyt Grant \# 1040794. This work was partially supported by ECOS Grant \# C02E08.

\section{References}

[1] A. Bensoussan, J.L. Lions, Applications of variational inequalities in stochastic control. Translated from the French. Studies in Mathematics and its Applications, 12. North-Holland Publishing Co., Amsterdam-New York, 1982.

[2] M. Bardi, F. Da Lio. Propagation of maxima and strong maximum principle for viscosity solutions of degenerate elliptic equation I: convex operators. Nonlinear Anal. 44 (2001), 991-1006.

[3] H. Berestycki, L. Nirenberg. On the method of moving planes and the sliding method, Boll. Soc. Brasil Mat. Nova ser. 22 (1991), 237-275.

[4] J. Busca, M. Esteban, A. Quaas. Nonlinear Eigenvalues and Bifurcation Problems for Pucci's Operator. Ann. Inst. H. Poincaré, Analyse non-linéaire 22 (2005), 187-206.

[5] X. Cabré, L. Caffarelli. Fully Nonlinear Elliptic Equation, American Mathematical Society, Colloquium Publication, Vol. 43, (1995).

[6] L. Caffarelli, B. Gidas, J. Spruck. Asymptotic symmetry and local behavior of semi-linear elliptic equations with critical Sobolev growth. Comm. Pure Appl. Math. 42, 3 (1989) 271-297. 
[7] C. Cerutti, L. Escauriaza, E. Fabes. Uniqueness for some diffusions with discontinuous coefficients. The Annals of Probability Vol.19, No2 (1991), 525537 .

[8] F. Da Lio, B. Sirakov. Symmetry results for viscosity solution of fully nonlinear elliptic equation, preprint.

[9] N. Dancer. The effect of domain shape on the number of positive solutions of certain nonlinear equations. Journal of Differential Equations 74,(1988), $120-156$.

[10] P. Felmer, A. Quaas. Critical Exponents for the Pucci's Extremal Operators, C.R. Acad. Sci. Paris (I) 335 (2002), pp. 909-914.

[11] P. Felmer, A. Quaas. On Critical exponents for the Pucci's extremal operators. Ann Inst. Henri Poicaré, Analyse non linéaire 20, no. 5 (2003), pp. 843-865.

[12] P. Felmer, A. Quaas. Positive solutions to 'semi-linear' equation involving the Pucci's operator, Journal of Differential Equations, 199, No 2,(2004), 376-393.

[13] D. Gilbarg, N. S. Trudinger. Elliptic partial differential equation of second order, 2nd ed., Springer-verlag (1983).

[14] N.V. Krylov. On one-point weak uniqueness for elliptic equations. Commun. in PDE, 17 (11\&12), (1992), 1759-1784.

[15] R. Jensen, M. Kocan and Swiech. Good and viscosity solutions of fully nonlinear elliptic equations. Proceedings of the American Mathematical Society, Vol. 130, No 2, (2001), 533-542.

[16] P.L. Lions. Optimal control of diffusion processes and Hamilton-JacobiBellman equations. I. The dynamic programming principle and applications. Comm. Partial Differential Equations 8, no. 10 (1983), pp. 1101-1174.

[17] P.L. Lions. Optimal control of diffusion processes and Hamilton-JacobiBellman equations. II. Viscosity solutions and uniqueness. Comm. Partial Differential Equations 8, no. 11 (1983), pp. 1229-1276.

[18] P.L. Lions. Optimal control of diffusion processes and Hamilton-JacobiBellman equations. III. Regularity of the optimal cost function. Nonlinear partial differential equations and their applications. Collège de France seminar, Vol. V (Paris, 1981/1982), pp. 95-205.

[19] C. Pucci. Maximum and minimum first eigenvalues for a class of elliptic operators. Proc. Amer. Math. Soc. 17, (1966), 788-795.

[20] C. Pucci. Operatori ellittici estremanti, Ann. Mat. Pure Appl. 72 (1966), $141-170$ 
[21] S.I. Pohozaev. Eigenfunctions of the equation $\Delta u+f(u)=0$. Soviet Math. 5 (1965), pp. 1408-1411.

[22] A. Quaas. Existence of positive solutions to a 'semi-linear' equation involving the Pucci's operator in a convex domain. Diff. Integral Equation, 17 (2004), 481-494.

[23] J. Smoller, G. Wasserman. Existence, uniqueness and non-degeneracy of positive solutions of semi-linear elliptic equations. Commun. Math. Phys. 95, 129-159 (1984).

E-mail addresses: esteban@ceremade.dauphine.fr,pfelmer@dim.uchile.cl, alexander.quaas@usm.cl 